\title{
Silanização de partículas de carga de compósitos odontológicos - revisão de literatura
}

\section{Silanization of filler particles of dental composites - literature review}

\author{
Lucielle Ferraz Guimarães* \\ Maranda de Oliveira** \\ Mariel Maas ${ }^{* *}$ \\ Nathália Ribeiro Andrade* \\ Luis Felipe Jochims Schneider** \\ Larissa Maria Cavalcante $e^{* * *}$
}

\section{Resumo}

Objetivo: o presente trabalho teve como objetivo realizar uma revisão de literatura com a intenção de simplificar o entendimento a respeito da importância do tipo de silano empregado e dos processos envolvidos na silanização de partículas de carga de compósitos odontológicos. Revisão de literatura: o agente silano é fundamental para promover a união entre as fases orgânica e inorgânica de compósitos odontológicos. No estudo, são abordados tópicos relacionados à formulação básica dos monômeros empregados nos compósitos odontológicos, às partículas de carga usadas, à importância do agente de união na composição do material, bem como aos tipos de silanos MPTS (y-metacrilioxipropil trimetoxisilano), OTMS (n-octiltrimetoxisilano), UDMS (3-[(1,3(2)-dimetacriloiloxipropil)-2(3)-oxicarbonillamido]propiltrietoxisilano), GPS (y-glicidoxipropil trimetoxisilano) e ATES (organosilanos allitrietoxisilano), efeitos das suas aplicações e dos processos de silanização. Considerações finais: a análise detalhada dos agentes silanos permite que o conhecimento das vantagens e desvantagens leve ao aprimoramento desses materiais e subsequente desenvolvimento de outros agentes de união mais apropriados à utilização na cavidade bucal.

Palavras-chave: Compósitos. Silano. Carga.

\section{Introdução}

Compósitos odontológicos são amplamente empregados como materiais restauradores diretos na clínica odontológica. Com uma notável propriedade mecânica e uma excelente característica estética, as resinas atuais são capazes de mimetizar propriedades singulares, dos dentes como cor, brilho e textura; entretanto o meio bucal ainda é adverso para a longevidade das restaurações. Interferências químicas, térmicas e mecânicas, atuando em diversas combinações, exercem papel fundamental no processo de degradação intraoral ao qual esses materiais restauradores são submetidos. Alterações na textura superficial e diminuição das propriedades físicas ao longo do tempo, causadas pelos processos de desgaste e de hidrólise no meio oral são fatores que indicam a necessidade do desenvolvimento de compósitos odontológicos com propriedades melhoradas e, consequentemente, mais duráveis. Visto isso, novos compósitos são frequentemente estudados e aperfeiçoados, tentando diminuir ou até mesmo cessar tais interferências nocivas do ambiente oral.

\footnotetext{
Cirurgiã-dentista, mestra em Dentística, Departamento de Odontotécnica, Universidade Federal Fluminense, Niterói, RJ, Brasil. Graduandas em Odontologia, Universidade Federal Fluminense, Niterói, RJ, Brasil.

Cirurgião-dentista, mestre e doutor em Materiais Dentários, Departamento de Odontologia Restauradora, Universidade Veiga de Almeida, Rio de Janeiro, RJ, Brasil e Departamento de Odontotécnica, Universidade Federal Fluminense, Niterói, RJ, Brasil.

**** Cirurgiã-dentista, mestra e doutora em Clínica Odontológica - Departamento de Odontotécnica, Úniversidade Federal Fluminense, Niterói, RJ, Brasil.
} 
Compreender os componentes utilizados para a formulação dos compósitos odontológicos torna-se imprescindível para a obtenção de materiais mais aprimorados e com melhorias importantes em suas características deficientes. Dessa forma, o objetivo do presente trabalho foi realizar uma revisão de literatura com a intenção de simplificar o entendimento a respeito da importância e dos processos envolvidos na silanização de partículas de carga de compósitos odontológicos. Os artigos foram selecionados a partir do site de busca Pubmed e tinham como tema a silanização de partículas de carga.

\section{Revisão de literatura}

\section{Matriz orgânica}

A matriz orgânica é constituída de monômeros, fotoiniciadores, coiniciadores, inibidores de polimerização, estabilizadores de UV e pequenas quantidades de componentes adicionais, que variam conforme $o$ fabricante $e^{1,2}$

O monômero mais utilizado nas formulações dos compósitos odontológicos é o dimetacrilato de bisfenol-A glicidila (Bis-GMA). Sua fórmula estrutural possui uma porção central epóxi e radicais acrílicos insaturados nas extremidades. Apresenta um alto peso molecular $(512 \mathrm{~g} / \mathrm{mol})$, alta viscosidade (500 000 - $800000 \mathrm{mPa}$ s), baixa mobilidade e baixo grau de conversão à temperatura ambiente ${ }^{3,4}$.

O trietilenoglicol dimetacrilato (TEGDMA) é menos viscoso (100 mPa:s) que o Bis-GMA e, portanto, é frequentemente utilizado como monômero diluente. A alta mobilidade do TEGDMA deve-se à sua estrutura de baixo peso molecular $(286 \mathrm{~g} / \mathrm{mol})$, e a adição deste resulta em compósitos com maior taxa de conversão ${ }^{5}$.

O Bis-GMA e o TEGDMA são os monômeros mais aplicados nas formulações dos compósitos odontológicos. ${ }^{2}$ Todavia, existem outros que também são utilizados, como o uretano dimetacrilatos (UDMA) e o bisfenol-A dimetacrilato etoxilado (BisEMA). O UDMA apresenta-se como base de alguns compósitos, isolado ou associado a outros monômeros. Seu peso molecular (470 g/mol) e sua viscosidade são considerados muito baixos (5 000 - $10000 \mathrm{mPa}$ ). Outro monômero que é adicionado em misturas dos compósitos consiste no BisEMA, que, diferentemente do Bis-GMA, não apresenta os grupos hidroxila pendentes que formam pontes de hidrogênio entre as moléculas. A viscosidade é menor quando comparado ao Bis-GMA, além de possuir alto peso molecular, $540 \mathrm{~g} / \mathrm{mol}^{4,6}$. Quanto menor a viscosidade da matriz orgânica, maior a possibilidade de incorporarem-se partículas de carga inorgânica ${ }^{4}$.

Sideridou et al. ${ }^{6}$ investigaram a influência da estrutura química no grau de conversão dos dimetacrilatos mais usados nas preparações de resinas compostas. Para tanto, utilizaram Bis-GMA, TEGDMA, BisEMA, UDMA e as misturas Bis-GMA / TEGDMA, Bis-GMA / UDMA e Bis-GMA / BisEMA, nas seguintes proporções: $35,8: 64,2$ e 56,6 : 43,4. O grau de conversão foi avaliado por espectrofotometria FT-IR, sendo registrado no tempo 0 e após cada período de fotoativação $(10 \mathrm{~s}, 20 \mathrm{~s}, 40 \mathrm{~s}, 60 \mathrm{~s}$, $80 \mathrm{~s}, 120 \mathrm{~s}, 180 \mathrm{~s}$ e $240 \mathrm{~s})$. Durante a fotoativação, foi utilizado um filme de polietileno que preveniu a inibição da polimerização pelo oxigênio. A temperatura de transição vítrea (Tg) foi utilizada para expressar a mobilidade do sistema durante a reação de polimerização, e o grau de conversão final das ligações duplas foi dependente da relação do grau de conversão com a $\mathrm{Tg}$ do sistema. Os resultados evidenciaram que o grau de conversão foi maior, na seguinte ordem: TEGDMA, UDMA, BisEMA e Bis-GMA. Os autores atribuíram a variação de comportamento à diferente estrutura química dos monômeros. O maior grau de conversão do TEGDMA, apesar da maior concentração de ligações duplas, foi relacionado ao fato de não ser viscoso e de possuir uma Tg muito baixa, o que conferiu maior mobilidade durante a polimerização. Já o resultado mostrado pelo UDMA foi maior que o esperado, apesar da alta $\mathrm{Tg}$, sendo atribuído ao fato de possuir o grupo - NH -, que favoreceu as reações de transferência de cadeia, aumentando a mobilidade dos locais dos radicais na rede e oferecendo caminhos alternativos para a continuação da reação de polimerização. O BisEMA teve o maior grau de conversão, em relação ao Bis-GMA, associado ao menor valor de Tg, que é atribuído à ausência dos grupos hidroxila pendentes, resultando em maior flexibilidade. E o pior resultado obtido pelo Bis-GMA deveu-se à presença do anel aromático rígido e às fortes pontes de hidrogênio que levam a um valor de $\mathrm{Tg}$ muito elevado. Em relação ao limite de grau de conversão obtido pelas misturas de Bis-GMA com dimetacrilatos, observou-se que há uma relação linear com a fração molar do Bis-GMA, além de uma relação com a $\mathrm{Tg}$ da mistura inicial de monômero, no caso da mistura Bis-GMA / TEGDMA.

\section{Partículas de carga inorgânica}

As partículas de carga inorgânica são utilizadas nos compósitos odontológicos com o objetivo principal de aumentar a resistência do material ${ }^{7,8}$. Materiais como quartzo, sílica coloidal, sílica de vidro contendo bário, estrôncio e zircônia e outros ${ }^{1,2}$ têm sido comumente utilizados nos compósitos como diferentes tipos de partículas de carga inorgânica. Diversos são os tipos, formas e tamanhos de carga utilizados em produtos comerciais, e esses fatores podem afetar as propriedades do material, como a dureza, ${ }^{9.10}$ o coeficiente de expansão térmica, ${ }^{11}$ a retenção de brilho, ${ }^{12}$ a absorção de água, ${ }^{13}$ a tenacidade à fratura ${ }^{14}$ e 0 módulo de elasticidade ${ }^{15,16}$. 
Dentro de cada tipo de compósito, os materiais distinguem-se pelas características de suas partículas de carga inorgânica e, em particular, pelo seu tamanho. Entretanto, a literatura relata que o tamanho e a área ocupada pela partícula da carga dentro da formulação de um compósito têm o potencial de influenciar no desempenho do desgaste, ou seja, quanto maior o tamanho da partícula, maior o desgaste ${ }^{17,18}$, levando a menor resistência à abrasão, maior rugosidade superficial, menor retenção de brilho e maior propensão ao manchamento superficial $^{19}$. Dessa forma, em razão do prejuízo estético observado ao longo do tempo, o uso desse tipo de compósito foi sendo substituído por meio do desenvolvimento de materiais com partículas em tamanhos menores ${ }^{16}$.

O tamanho de partículas em compósitos considerado médio é de $0,02 \mu \mathrm{m}$ (com variação de 0,01 $0,05 \mu \mathrm{m}$ ). São classificados como microparticulados e foram desenvolvidos objetivando melhorar a estética ${ }^{20,21}$. Entretanto, as propriedades mecânicas desses materiais não são consideradas adequadas para o uso em áreas de grande esforço mastigatório ${ }^{22}$. Essa característica é atribuída a menor quantidade de partícula de carga inorgânica e, consequentemente, maior quantidade de matriz orgânica ${ }^{23,24}$.

Os compósitos híbridos foram desenvolvidos no sentido de melhorar as propriedades mecânicas pela incorporação de partículas de carga inorgânica, composta por diferentes tamanhos médios de partículas, $15-20 \mu \mathrm{m}$ e $0,01-0,05 \mu \mathrm{m}^{20}$. Já os compósitos micro-híbridos foram formulados com a intenção de melhorar a capacidade de polimento da superfície dos materiais, diminuindo o tamanho médio das partículas com menos de $1 \mu \mathrm{m}$ e uma variação de $0,1-0,6 \mu \mathrm{m}^{16,22}$.

O acabamento e polimento são passos importantes que favorecem a estética e a longevidade dos compósitos $^{25,26}$. A rugosidade superficial, associada com o acabamento e polimento inadequados, pode resultar em maiores taxas de desgaste e acúmulo de placa bacteriana. Isso compromete o desempenho clínico da restauração ${ }^{27}$.

Uma modificação importante no conteúdo inorgânico realizada recentemente é a aplicação da nanotecnologia à formulação dos compósitos odontológicos. Partículas de carga inorgânica em escala nanométrica (1 - $100 \mathrm{~nm})$ foram incorporadas à matriz orgânica com o objetivo principal de melhorar a textura superficial e as propriedades óticas desses materiais ${ }^{16,28}$.

Curtis et al. ${ }^{29}$ verificaram a influência das partículas de carga inorgânica de tamanho nanométrico e aglomerados de nanopartículas (nanoclusters) em compósitos comerciais por meio da resistência à flexão biaxial e do armazenamento em ambiente seco e imerso em água. Os autores observaram que o aglomerado é um mecanismo de reforço distinto em comparação com os compósitos micro-híbridos, microparticulados e nano-híbridos, resultando em melhoras significativas na resistência à flexão, independentemente das condições de armazenamento. As nanopartículas aglomeradas produziram uma rede interligada, na qual os interstícios foram infiltrados com o agente de união silano, produzindo uma estrutura composta de fases interpenetrantes. A combinação desse reforço com a infiltração do agente de união silano nas porosidades estruturais melhorou a resistência à flexão e aumentou a longevidade clínica das restaurações.

Para que as partículas de carga inorgânica possam atuar, de modo efetivo, no aumento da resistência do compósito, é necessário que estejam quimicamente unidas à matriz orgânica ${ }^{19,30,31}$. Assim, a formação de uma forte ligação covalente entre a partícula de carga inorgânica e a matriz orgânica é essencial para obter-se boas propriedades mecânicas nos compósitos odontológicos, e a união dessas duas fases é alcançada pelo revestimento da partícula de carga inorgânica com um agente de união silano denominada de "interface" $1,32,33$.

Materiais formulados com partículas nanométricas podem trazer benefícios como melhora da lisura superficial, ${ }^{12}$ excelente resistência mecânica ao desgaste, máxima estética devido à maior fração volumétrica de cargas e à facilidade na manutenção do polimento ${ }^{12}$. Especificamente no caso desses materiais, o agente de união silano pode apresentar uma função ainda mais importante. Em virtude da ampla relação área de superfície / volume disponível por essas partículas, é necessário o uso de uma quantidade superior de agente de união silano, quando comparado com as partículas tradicionalmente usadas (em escala micrométrica). Essa quantidade de silano pode interferir em diversas propriedades físico-químicas dos materiais formulados.

\section{Agente de união - silano}

$\mathrm{O}$ agente silano apresenta uma importante função como promotor da união entre as fases orgânica e inorgânica na formulação de compósitos odontológicos. A silanização do conteúdo inorgânico promove melhores propriedades mecânicas, tais como resistência à flexão e à tração, tenacidade à fratura e módulo de elasticidade ${ }^{33,34}$. Além disso, a presença do agente de união silano parece reduzir a degradação por hidrólise, evitando a penetração de água na interface carga / matriz, quando comparado com compósitos formulados sem o uso do silano ${ }^{35}$. Esse fato pode ser atribuído à união química promovida pelo silano entre partículas de carga inorgânica com a matriz orgânica ${ }^{36}$.

Diversos tipos de silanos podem ser utilizados na formulação dos compósitos odontológicos. Destes, os mais estudados são o MPTS ( $\gamma$-metacriliox ipropiltrimetoxisilano), o OTMS (n-octiltrimetoxisilano), o UDMS (3-[(1,3(2)-dimetacriloiloxipropil)- 
-2(3)-oxicarbonillamido]propiltrietoxisilano), o GPS ( $y$-glicidoxipropil trimetoxisilano) e o ATES (organosilanos allitrietoxisilano $)^{35}$.

O silano mais frequentemente utilizado na formulação de compósitos odontológicos é o MPTS (Figura 1). Além de evitar a agregação das partículas de carga, o MPTS promove uma união entre as partículas de carga inorgânica e a matriz orgânica, por meio da copolimerização, mediante ligações covalentes e pontes de hidrogênio ${ }^{33,36}$.

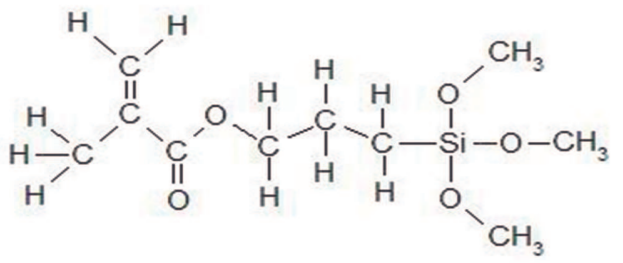

MPTS

Figura 1 -Silano MPTS.

Em contrapartida, o agente OTMS (Figura 2) é um silano alifático não reativo com a matriz orgânica e que interage quimicamente por meio de fracas forças de Van der Walls ${ }^{1,35}$. A porção orgânica presente no agente OTMS diferencia-se do MPTS, de forma a não reagir por meio de ligações covalentes com a matriz orgânica durante o processo de polimerização do material ${ }^{34}$. Ausência de grupos metacrilatos no agente OTMS impossibilita que ocorra uma reação de união adequada entre matriz orgânica e partícula de carga inorgânica, o que pode contribuir com o processo de hidrólise e desgaste ${ }^{33}$.

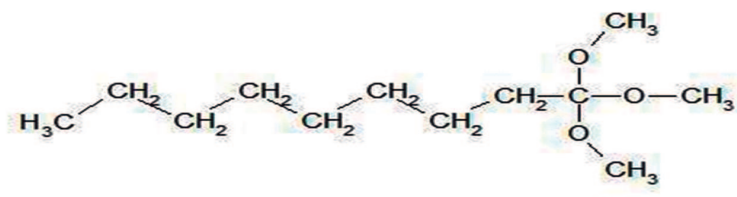

OTMS

Figura 2 - Silano OTMS

Wilson et al..$^{33}$ (2005) determinaram o efeito da variação química da interface nas propriedades dos nanocompósitos. Nanopartículas de sílica foram silanizadas com dois tipos de silano (MPTS e OTMS), mantendo a quantidade total de silano constante em $10 \%$ pela fração de massa relativa à massa da partícula de carga inorgânica, nas seguintes proporções de MPTS / OTMS (10 / 0; 7,5 / 2,5; 5 / 5; $2,5 / 7,5 ; 0 / 10)$. As nanopartículas silanizadas foram adicionadas a uma resina de dimetacrilato. As propriedades mecânicas dos compósitos não fotoativados foram avaliadas por testes de compressão.
Compósitos fotoativados foram testados quanto à resistência à flexão biaxial e de três pontos. As características de manipulação dos compósitos com maiores quantidades de OTMS foram melhores em comparação às maiores concentrações de MPTS. A resistência à flexão do MPTS silanizado e dos compósitos duais silanizados foi semelhante, mas diminuiu quando frações de massa OTMS aumentaram para 7,5 e $10 \%$. Os autores constataram que, nas propriedades mecânicas, as concentrações de 7,5 MPTS / 2,5 OTMS e 5 MPTS / 5 OTMS dos compósitos foram comparáveis às dos compósitos com 10 MPTS. A química de superfície otimizada irá permitir maiores frações de massa da fase de carga e, por isso, pode reduzir a contração de polimerização em comparação com os compósitos híbridos contendo apenas partículas de carga silanizadas com MPTS. Além disso, as partículas de silanização dupla têm o potencial de alterar as propriedades da interface, por exemplo, pela redução de absorção de água em razão do aumento da hidrofobicidade derivada do hidrocarboneto octil silano, e também, possivelmente, reduzindo o estresse de polimerização por causa de uma maior flexibilidade do silano OTMS comparado com o MPTS.

O MPTS é uma molécula bifuncional que apresenta grupos de silanóis ( $\mathrm{Si}-\mathrm{OH}$ ) de um lado e grupos de metacrilatos (contendo $\mathrm{C}=\mathrm{C}$ ) na outra extremidade ${ }^{3}$. Para ser capaz de agir como agente de união, primeiramente o silano deve ser hidrolizado (ativado) e, posteriormente, sofrer uma reação de condensação, na qual duas moléculas combinam-se para formarem uma só molécula. Durante a reação, libera-se a menor molécula, neste caso, a de água ${ }^{36}$.

$\mathrm{O}$ grupo funcional metoxi $\left[-\mathrm{Si}\left(\mathrm{OCH}_{3}\right)_{3}\right]$ do silano, hidrolisado na presença de água, produz grupos silânicos reativos, - $\mathrm{Si}(\mathrm{OH})_{3}$ (Figura 3). Estes aderem aos grupos silanóis na superfície da sílica, por meio da hidroxila $(\mathrm{OH})$, formando pontes de hidrogênio. $\mathrm{O}$ grupo carbonil $(\mathrm{C}=\mathrm{O})$ do silano também forma pontes de hidrogênio com a hidroxila da superfície da sílica $^{37}$.

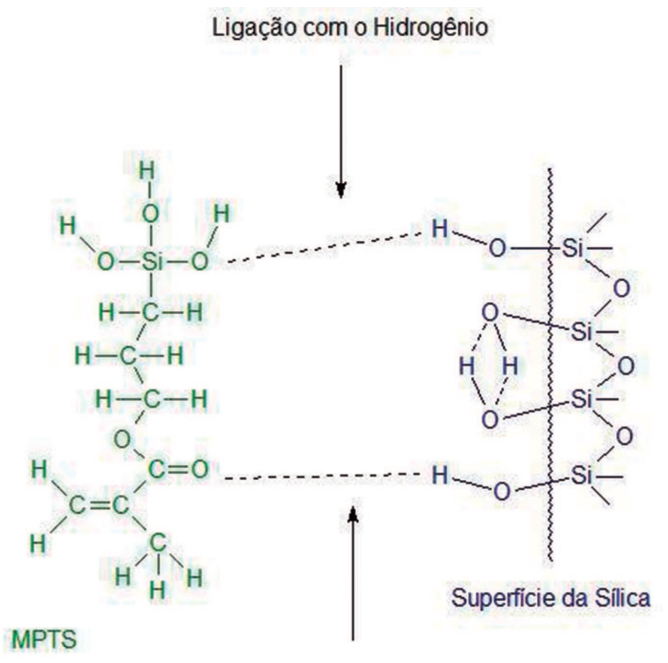

Ligação com o Hidrogênio 
A interação do grupo silanol do silano com a hidroxila da sílica resulta na reação de condensação, formando uma ligação covalente entre a molécula do silano e a superfície da sílica, Si-O-Si (Figura 4 e 5). Com isso, a partícula de carga inorgânica é coberta por várias moléculas de silano, tornando a interface complexa ${ }^{38}$. Já o grupo metacrilato $(\mathrm{C}=\mathrm{C})$ forma ligação covalente com a matriz orgânica, finalizando o processo de união $0^{39,40}$.

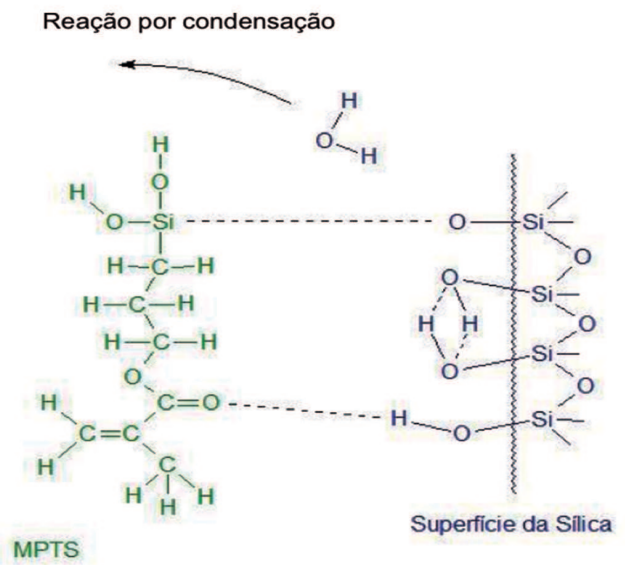

Figura 4 - Reação de união do silano com a partícula de carga inorgânica

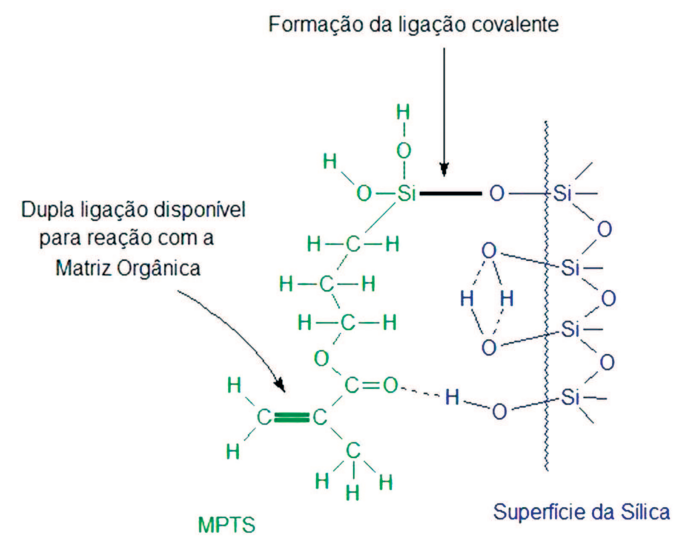

Figura 5 - Reação de união do silano com a partícula de carga inorgânica e a matriz orgânica

Sideridou e Karabela ${ }^{35}$ avaliaram o efeito da quantidade de silano MPTS (3-methacril-oxipropil-trimethoxisilano) nas propriedades físico-mecânicas de um compósito experimental. Nanopartículas de sílica (Aerosil OX 50) foram silanizadas ${ }^{41} \mathrm{com}$ diferentes quantidades de silano MPTS: 1,0, 2,5, 5,0, 7,5 e $10 \%$ em peso em relação à sílica e, em seguida, adicionadas aos monômeros Bis-GMA / TEGDMA (50 / 50\% em peso). Não foi observada correlação com a variação na quantidade de silano utilizado e o grau de conversão. Em relação às propriedades mecânicas, resistência à flexão e módulo de flexibilidade, não houve diferença estatística significante com diferentes concentrações de silano. Em quantidades mais elevadas de silano MPTS, o módulo de elasticidade diminuiu. Já os compósitos com maiores quantidades de silano MPTS mostraram valores mais baixos para Tan e Tg, revelando uma melhor adesão interfacial entre a matriz e partícula de carga inorgânica. A absorção, a solubilidade e a alteração volumétrica foram determinadas após o armazenamento dos compósitos em solução de água ou etanol/ água. Quanto menor a quantidade de silano MPTS, maior a quantidade de água absorvida. Ao comparar a quantidade de água absorvida e não absorvida com a quantidade de solução de etanol / água, os autores observaram para todos os compósitos que a quantidade de solução de etanol / água absorvida e não absorvida é muito maior do que a de água.

Karmaker et al. ${ }^{42}$ avaliaram o efeito de diferentes concentrações de silano $(0,75,1,5,2,0,3,0,6,0$, 8,0 e $14 \%$ em peso) nos compósitos experimentais por meio da viscosidade, resistência à flexão e à degradação hidrolítica. As partículas de carga consistiam da mistura de vidro de bário e sílica amorfa, e a resina foi baseada de Bis-GMA e dimetacrilato policarbonato. A adsorção do silano na superfície da partícula de carga inorgânica foi caracterizada por FT-IR. O comportamento reológico dos compósitos foi determinado por um viscosímetro. As viscosidades dos compósitos contendo 1,5\% em peso de silano e não silanizados foram semelhantes e superiores às dos demais grupos. O aumento na concentração do silano (MPTS) levou a uma diminuição na viscosidade dos compósitos experimentais. Os compósitos com cargas silanizadas obtiveram maior resistência à flexão quando comparados com as partículas de carga não silanizadas. No entanto, nenhuma diferença significativa foi encontrada entre os grupos silanizados. A resistência à degradação por hidrólise dos diferentes compósitos aumentou quando as partículas de carga foram tratadas com silano e foi o maior em 3,0\% em peso de silano. Concluiu-se que o tratamento das partículas de carga inorgânica com silano MPTS, em geral, melhorou tanto na resistência à flexão dos compósitos quanto na resistência à degradação por hidrólise.

\section{Considerações finais}

A quantidade de agente de união silano utilizada na formulação dos compósitos pode interferir nas propriedades do material. Dessa forma, uma influência parece existir entre as propriedades físico-químicas dos compósitos e a quantidade de silano utilizada na cobertura das partículas de carga inorgânica na formulação de compósitos odontológicos. 


\section{Abstract}

Objective: this study aimed to perform a literature review to facilitate the understanding regarding the type of silane used, as well as the processes involved in silanization of filler particles of dental composites. Literature review: the silane agent is essential to promote a bond between organic and inorganic phases of dental composites. The topics addressed in this study are: basic formulation of monomers employed in dental composites, filler particles used, the importance of the bonding agent in material composition, as well as the types of MPTS silane ( $y$ - methacryloxypropyltrimethoxysilane), OTMS (n-octyltrimethoxysilane) UDMS (3-[(1,3(2)-dimethacryloyloxypropyl)-2 (3)-oxycarbonylamido] propyl triethoxysilane), GPS (y- glycidoxypropyltrimethoxysilane), and ATES (organosilanes alyltriethoxysilane), the effects of its applications and silanization processes. Final considerations: a detailed analysis of silane agents allows the knowledge of the advantages and disadvantages of these materials to lead to the improvement and subsequent development of other bonding agents that are more suitable for use in the oral cavity.

Keywords: Composite material. Silane. Fillers.

\section{Referências}

1. Chen MH. Update on dental nanocomposites. J Dent Res 2010; 89(6):549-60.

2. Schneider LFJ, Cavalcante LM, Silikas N. Shrinkage stresses generated during resin-composite applications: a review. J Dent Biomech 2010: 131630 .

3. Ferracane JL. Current trends in dental composites. Crit Rev Oral Biol Med 1995; 6:302-18.

4. Peutzfeldt A. Resin composites in dentistry: the monomer systems. Eur J Oral Sci 1997; 105(2):97-116

5. Asmussen E, Peutzfeldt A. Influence of pulse-delay curing on softening of polymer structures. J Dent Res 2001; 80(6):1570-3.

6. Sideridou I, Tserki V, Papanastasiou G. Effect of chemical structure on degree of conversion in light-cured dimethacrylate-based dental resins. Biomaterials 2002; 23:1819-29.

7. Ferracane JL, Antonio RC, Matsumoto H. Variables affecting the fracture toughness of dental composites. J Dent Res 1987; 66(6):1140-5.

8. Wilson KS, Antonucci JM. Interphase structure-property relationships in thermoset dimethacrylate nanocomposites. Dent Mater 2006; 22(11):995-1001.

9. Braem M, Finger W, Van Doren VE, Lambrechts P, Vanherle G. Mechanical properties and filler fraction of dental composites. Dent Mater 1989; 5(5):346-9.

10. Kim KH, Park JH, Imai Y, Kishi T. Microfracture mechanisms of dental resin composites containing spherically-shaped filler particles. J Dent Res 1994; 73(2):499-504.

11. Soderholm KJ. Influence of silane treatment and filler fraction on thermal expansion of composite resins. J Dent Res 1984; 63(11):1321-6.
12. Cavalcante LM, Masouras K, Watts DC, Pimenta LA, Silikas N. Effect of nanofillers' size on surface properties after toothbrush abrasion. Am J Dent 2009; 22(1):60-4.

13. Baroudi K, Silikas N, Watts DC. Time-dependent visco-elastic creep and recovery of flowable composites. Eur J Oral Sci 2007; 115(6):517-21.

14. Kim KH, Ong JL, Okuno O. The effect of filler loading and morphology on the mechanical properties of contemporary composites. J Prosthet Dent 2002; 87(6):642-9.

15. Masouras K, Silikas N, Watts DC. Correlation of filler content and elastic properties of resin-composites. Dent Mater $2008 ; 24(7): 932-9$.

16. Ferracane JL. Review resin composite - State of the art. Dent Mater 2011; 27:29-38.

17. Crumpler DC, Heymann HO, Shugars DA, Bayne SC, Leinfelder KF. Five-year clinical investigation of one conventional composite and three microfilled resins in anterior teeth. Dent Mater 1988; 4(4):217-22.

18. Roulet JF. A materials scientist's view: assessment of wear and marginal integrity. Quint Int 1987; 18:543-52.

19. Turssi CP, Ferracane JL, Vogel K. Filler features and their effects on wear and degree of conversion of particulate dental resin composites. Biomaterials 2005; 26:4932-7.

20. Lutz F, Phillips RW. A classification and evaluation of composite resin systems. J Prosthet Dent 1983; 50(4):480-8.

21. Van Noort R. Introduction to dental materials. 3. ed. London: Elsevier; 2007.

22. Bayne SC, Heymann HO, Swift Jr EJ. Update on dental composite restorations. J Am Dent Assoc 1994; 125:687-701.

23. Pallav P, De Gee AJ, Davidson CL, Erickson RL, Glasspoole EA. The influence of admixing microfiller to small-particle composite resin on wear, tensile strength, hardness, and surface roughness. J Dent Res 1989; 68:489-90.

24. Soderholm KJ. Degradation of glass filler in experimental composites. J Dent Res 1981; 60:1867-75.

25. Goldstein RE. Finishing of composites and laminates. Dent Clin North Am 1989; 33:305-18.

26. Jefferies SR. The art and science of abrasive finishing and polishing in restorative dentistry. Dent Clin North Am 1998; 42:613-27.

27. Bollen CML, Lambrechts P, Quirynen M. Comparison of surface roughness of oral hard materials to the threshold surface roughness for bacterial plaque retention: a review of the literature. Dent Mater 1997; 13(4):258-69.

28. Mitra SB, Wu D, Holmes BN. An application of nanotechnology in advanced dental materials. J Am Dent Assoc 2003; 134(10):1382-9.

29. Curtis AR, Palin WM, Fleming GJ, Shortall AC, Marquis PM. The mechanical properties of nanofilled resin-based composites: Characterizing discrete filler particles and agglomerates using a micromanipulation technique. Dent Mater 2009; 25:180-7.

30. Arksornnukit M, Takahashi H, Nishiyama N, Pavasant P. Effects of heat and $\mathrm{pH}$ in silanation process on flexural properties and hydrolytic durabilities of composite resin after hot water storage. Dent Mater J 2004; 23(2):175-9. 
31. Musanje L, Ferracane JL, Ferracane LL. Effects of resin formulation and nanofiller surface treatment on in vitro wear of experimental hybrid resin composite. J Biomed Mater Res B Appl Biomater 2006; 77:120-5.

32. García AH, Lozano MAM, Vila JC, Escribano AB, Galve PF. Composite resins. A review of the materials and clinical indications. Med Oral Patol Oral Cir Bucal 2006; 11:E215-20.

33. Wilson KS, Zhang K, Antonucci JM. Systematic variation of interfacial phase reactivity in dental nanocomposites. Biomaterials 2005; 26(25):5095-103.

34. Wilson KS, Allen AJ, Washburn NR, Antonucci JM. Interphase effects in dental nanocomposites investigated by small-angle neutron scattering. J Biomed Mater Res A 2007; 81(1):113-23.

35. Sideridou ID, Karabela MM. Effect of the amount of 3-methacyloxypropyltrimethoxysilane coupling agent on physical properties of dental resin nanocomposites. Dent Mater 2009; 25(11):1315-24.

36. Matinlinna JP, Lassila LVJ, Vallittu PK. The effect of five silane coupling agents on the bond strength of a luting cement to a silica-coated titanium. Dent Mater 2007; 2(3):1173-80.

37. Plueddemann EP. Silane coupling agent. New York: Plenum; 1991. p. 31-54.

38. Soderholm KJM, Shang SW. Molecular-orientation of silane at the surface of colloidal silica. J Dent Res 1993; $72(6): 1050-4$.

39. Nihei T, Dabanoglu A, Teranaka T, Kurata S, Ohashi K, Kondo Y, et al. Three-body-wear resistance of the experimental composites containing filler treated with hydrophobic silane coupling agents. Dent Mater 2008; 24(6):760-4.

40. Shen CY, Oh WS, Williams JR. Effect of post-silanization drying on the bond strength of composite to ceramic. J Prosthet Dent 2004; 91(5):453-8.

41. Chen TM, Brauer GM. Solvent effects on bonding organo-silane to sílica surfaces. J Dent Res 1982; 61:1439-43.

42. Karmaker A, Prasad A, Sarkar NK. Characterization of adsorbed silane on fillers used in dental composite restoratives and its effect on composite properties. J Mater Sci Mater Med 2007; 18(6):1157-62.

Endereço para correspondência:

Larissa Maria Cavalcante

Faculdade de Odontologia da UFF

Rua Mário Santos Braga, 30 - 3oandar

24020-140 Niterói, RJ

Fone: (21) 81033636

E-mail: lara_cavalcante@yahoo.com.br 